\title{
Regulation of UDP-Glucuronosyltransferases UGT2B4 and UGT2B7 by MicroRNAs in Liver Cancer Cells ${ }^{[\mathbf{S}}$
}

\author{
Dhilushi D. Wijayakumara, Peter I. Mackenzie, Ross A. McKinnon, Dong Gui Hu, \\ and Robyn Meech
}

Department of Clinical Pharmacology and Flinders Centre for Innovation in Cancer, Flinders University School of Medicine, Flinders Medical Centre, Bedford Park, South Australia, Australia

Received December 21, 2016; accepted February 21, 2017

\begin{abstract}
The transcriptional regulation of UDP-glucuronosyltransferases UGT2B4 and UGT2B7 has been well studied using liver cancer cell lines, and post-transcriptional regulation of these two UGTS by microRNA (miRNA/miR) miR-216b-5p was recently reported. This study describes novel miRNA-mediated regulation of UGT2B4 and UGT2B7 in liver cancer cells. Bioinformatic analyses identified a putative miR-3664-3p binding site in the UGT2B7 3'-untranslated region (UTR) and binding sites for both miR-135a-5p and miR-410-3p in the UGT2B4 $3^{\prime}$-UTR. These sites were functionally characterized using miRNA mimics and reporter constructs. A miR-3664-3p mimic induced repression of a luciferase reporter carrying the UGT2B7 3'-UTR in liver cancer cell lines; mutation of the miR-3664-3p site abrogated the response of the reporter to the mimic. Similarly, mutation of the miR-135a-5p site or miR-410-3p site in a luciferase reporter
\end{abstract}

bearing UGT2B4 $3^{\prime}$-UTR abrogated the ability of miR-135a-5p or miR-410-3p mimics to reduce reporter activity. Transfection of miR-3664-3p mimics in HepG2 liver cancer cells significantly reduced mRNA and protein levels of UGT2B7, and this led to reduced enzymatic activity. Transfection of miR-135a-5p or miR410-3p mimics significantly decreased UGT2B4 mRNA levels in Huh7 liver cancer cells. The expression levels of miR-410-3p were inversely correlated with UGT2B4 mRNA levels in The Cancer Genome Atlas cohort of liver hepatocellular carcinoma (371 specimens) and a panel of ten normal human tissues. Similarly, there was an inverse correlation between miR-135a and UGT2B4 mRNA levels in a panel of 18 normal human liver tissues. Together, these data suggest that miR-135a and miR410 control UGT2B4 and that miR-3664 controls UGT2B7 expression in liver cancer and/or normal liver cells.

\section{Introduction}

Glucuronidation refers to the covalent attachment of glucuronic acid to a variety of endogenous and exogenous small lipophilic compounds; glucuronidated products are generally biologically inactive and more water soluble, thus facilitating their excretion from the body (Mackenzie et al., 1997, 2005). There are four human UDP-glucuronosyltransferase (UGT) families (Mackenzie et al., 2005); however, glucuronidation is primarily carried out by members of the UGT1A and UGT2B subfamilies. Substrates for these enzymes include endogenous bioactive molecules (e.g., steroids, thyroid hormones, bilirubin, bile acids, retinoids, and fatty acids), carcinogens, environmental pollutants, and therapeutic drugs (Guillemette,

This research was supported by the National Health and Medical Research Council (NHMRC) of Australia [Grants ID1020931 (to P.I.M.) and ID1085410 (to P.I.M., R.A.M., and R.M.)]. The project was also supported by funding from the Flinders Medical Centre Foundation. R.A.M. is a Cancer Council/SA Health Beat Cancer Professorial Chair. During the preparation period, P.I.M. was an NHMRC Senior Principal Research Fellow; R.M. was an Australian Research Council Future Fellow.

D.G.H. and R.M. are co-last authors.

https://doi.org/10.1124/jpet.116.239707.

S This article has supplemental material available at jpet.aspetjournals.org.
2003; Mackenzie et al., 2005). It was estimated that UGTs are involved in the metabolism of $35 \%$ of clinically administered drugs (Guillemette, 2003).

The liver is the primary site of glucuronidation; thus, the control of hepatic UGT expression and activity is of significant biologic and pharmacological interest (Hu et al., 2014b). UGT2B4 and UGT2B7 are both highly expressed in the liver (Congiu et al., 2002; Court, 2010). UGT2B7 has high activity toward various endogenous compounds, including bile acids, retinoic acids, steroids, mineralocorticoid and glucocorticoid hormones, and fatty acids (Hu et al., 2014b), as well as exogenous compounds, including carcinogens, therapeutic drugs such as morphine, codeine (Coffman et al., 1997), epirubicin (Innocenti et al., 2001), valproic acid (Argikar and Remmel, 2009), and nonsteroidal anti-inflammatory drugs (Jin et al., 1993). Around 35\% of the therapeutic drugs that are glucuronidated by UGTs are UGT2B7 substrates (Williams et al., 2004). In addition to hepatic expression, UGT2B7 is expressed in the small intestine, colon, kidney, and breast, where it may also play important roles in drug metabolism and homeostasis of endogenous bioactive molecules (Ohno and Nakajin, 2009; Hu et al., 2014b). UGT2B4 is also abundantly expressed in many extrahepatic tissues and organs

ABBREVIATIONS: GAPDH, glyceraldehyde-3-phosphate dehydrogenase; HNF, hepatocyte nuclear factor; HPLC, high-performance liquid chromatography; LIHC, liver hepatocellular carcinoma; miR, microRNA; miR-neg, negative control miRNA; miRNA, microRNA; PCR, polymerase chain reaction; RNAseq, RNA sequencing; RT-q, reverse-transcriptase quantitative real-time; TCGA, The Cancer Genome Atlas; UGT, UDPglucuronosyltransferase; UTR, untranslated region. 
(e.g., kidney and esophagus) (Ohno and Nakajin, 2009) and has an important role in the glucuronidation of bile acids (FournelGigleux et al., 1989), catechol-estrogens (Ritter et al., 1992), androgen metabolites (Lévesque et al., 1999; Turgeon et al., 2001; Lépine et al., 2004), and phenols (Guillemette, 2003). UGT2B4 is involved in the glucuronidation of hydroxylated bile acids (Barre et al., 2007), as well as a subset of drugs and toxins including deoxynivalenol (a mycotoxin) (Maul et al., 2015), hydroxymidazolam (Seo et al., 2010), lorazepam (Uchaipichat et al., 2013), codeine (Raungrut et al., 2010), eslicarbazepine (an antiepileptic) (Loureiro et al., 2011), and carvedilol (a $\beta$-adrenoceptor blocker) (Ohno et al., 2004). However, in general, UGT2B4 is considered to play a more minor role in overall drug metabolism than UGT2B7.

As recently reviewed (Hu et al., 2014b), the transcriptional regulation of UGT2B4 and UGT2B7 has been well investigated using hepatic models such as liver cancer cell lines, human primary hepatocytes, and transgenic mice. The transcription factors found to be involved in controlling UGT2B7 expression include hepatocyte nuclear factor (HNF) $1 \alpha$ (Ishii et al., 2000), HNF $4 \alpha$, caudal related homeobox factor 2 (Cdx2) (Gregory et al., 2006), nuclear factor (erythroid-derived 2)-like 2 (Nrf2) (Nakamura et al., 2008), farnesoid X receptor (Lu et al., 2005), p53 (Hu et al., 2014c), and activator protein 1 (Hu et al., 2014a). UGT2B4 is known to be transcriptionally regulated by the farnesoid $\mathrm{X}$ receptor, retinoid $\mathrm{X}$ receptor, and peroxisome proliferator-activated receptor $\alpha$ (Barbier et al., 2003a,b). MicroRNAs (miRNAs/miRs) are noncoding small RNAs (approximately 21-25 nucleotides) that mediate post-transcriptional gene regulation through translational repression and/or mRNA degradation (Macfarlane and Murphy, 2010). The first identification of a miRNA involved in UGT2B4 and UGT2B7 regulation (miR-216b-5p) in liver cancer cell lines was recently reported (Dluzen et al., 2016). Our study provides in-depth characterization of the roles of three additional miRNAs (miR-3664-3p, miR-135a-5p, and miR-410-3p) in the regulation of UGT2B4 and UGT2B7 in liver cancer cell lines.

\section{Materials and Methods}

miRNA Mimics and Human Tissue Total RNA. Mimics of hsa-miR3664-3p, hsa-miR-135a-5p, hsa-miR-410-3p, hsa-miR-1266-5p, hsa-miR-4483, hsa-miR-4317, hsa-miR216b-5p,has-miR-489-3p, hsamiR-4691-5p, hsa-miR-101-3p, and a negative control miRNA (termed miR-neg) were purchased from Shanghai GenePharma (Shanghai, China). A miRNA inhibitor of miR-3664-3p and a negative control were purchased from Ambion/Applied Biosystems (Foster City, CA). Total RNA samples from a panel of human tissues were purchased from Ambion/Applied Biosystems (FirstChoice Human Total RNA Survey Panel). The normal human liver tissues were previously reported (Hu et al., 2014a).

Cell Transfection, RNA Extraction, and Reverse-Transcriptase Quantitative Real-Time Polymerase Chain Reaction. HepG2 cells were obtained from the American Type Culture Collection (Manassas, VA) and HuH7 cells were obtained from Dr. Jillian Carr (Flinders University, Adelaide, SA, Australia). Both cell lines were maintained in Dulbecco's modified Eagle's medium containing $10 \%$ $(\mathrm{v} / \mathrm{v})$ fetal bovine serum at $37^{\circ} \mathrm{C}$ in a $5 \% \mathrm{CO}_{2}$ atmosphere. Cells were plated in six-well plates and cultured overnight, reaching 60\%$70 \%$ confluence prior to transfection. Transfections were performed with miRNA mimics, miRNA inhibitor, or miR-neg in triplicate at $30 \mathrm{nM}$ using $8 \mu \mathrm{l}$ Lipofectamine 2000 (Invitrogen, Carlsbad, CA) per well. Total RNA was extracted after 24 hours using TRIzol reagent according to the manufacturer's protocol (Invitrogen). The expression levels of target genes [UGT2B7, UGT2B4, 18S rRNA, and glyceraldehyde-3-phosphate dehydrogenase $(G A P D H)]$ were quantified using reverse-transcriptase quantitative real-time (RT-q) polymerase chain reaction (PCR) in a RotorGene 3000 instrument (Corbett Research, Mortlake, NSW, Australia) as previously reported (Hu and Mackenzie, 2009, 2010; Hu et al., 2010). Quantification of miRNAs (miR3664-3p, miR-135a-5p, miR-410-3p miR-1266-5p, miR-4483, miR-4317, miR216b-5p, miR-489-3p, miR-4691-5p, miR-101-3p) and RNU6-2 (miR-U6 small nuclear-2 RNA as a housekeeping miRNA) was performed as previously reported (Balcells et al., 2011; Wijayakumara et al., 2015). The expression levels of target genes relative to $18 \mathrm{~S}$ rRNA were quantified using the 2(-Delta Delta $\mathrm{C}(\mathrm{T})$ ) method (Livak and Schmittgen, 2001). The expression levels of individual miRNAs were presented relative to that of RNU6-2 (set as a value of $100 \%$ ). Primers for RT-qPCR of miRNAs are listed in Table 1.

Generation of Luciferase Reporter Constructs and Mutagenesis. The UGT2B7 mRNA (NM_001074.3) contains a 251-nucleotide 3'untranslated region (UTR), whereas the UGT2B4 mRNA(NM_021139.2) contains a 469-nucleotide 3'-UTR. The full-length UGT2B7 3'-UTR region between the stop codon (TAG) and the poly(A) tail was amplified from human genomic DNA (Roche Diagnostics, Indianapolis, IN) using Phusion Hot-Start High-Fidelity DNA Polymerase (Thermo Fisher Scientific, Pittsburgh, PA) and cloned into the $\mathrm{XbaI}$ restriction site of the pGL3-promoter vector (Promega, Madison, WI), generating the reporter construct pGL3/2B7/UTR. Similarly, the full-length UGT2B4 3 '-UTR region between the stop codon and the poly(A) tail was amplified and cloned into the pGL3-promoter vector, generating the reporter construct pGL3/2B4/UTR. Primers used for amplifying the $3^{\prime}$-UTR of UGT2B4 or UGT2B7 are listed in Table 1.

Using the pGL3/2B7/UTR construct as a template and the QuickChange site-directed mutagenesis kit (Stratagene, La Jolla, CA), the UGT2B7 miR-3664-3p seed site ( $5^{\prime}$-UCCUGAG- $3^{\prime}$ ), which is complementary to the miR-3664-3p seed sequence ( $3^{\prime}$-AGGACUC-5'), was changed to $5^{\prime}$-UAGCUAG- $3^{\prime}$, producing the mutated construct pGL3/2B7/UTR/miR-3664/MT. Similarly, using the pGL3/2B4/UTR construct as a template, the UGT2B4 miR-135a-5p seed site was mutated from 5'-AAAGCCAU-3' to 5'-AAAUGACU-3', generating the mutated construct pGL3/2B4/UTR/miR-135a/MT; the UGT2B4 miR-410-3p seed site was mutated from $5^{\prime}$-GUUAUAU-3' to $5^{\prime}$ GUCCGCU-3', generating the mutated construct pGL3/2B4/UTR/ miR-410/MT. The identities of all constructs were confirmed by DNA sequencing. Primers used for mutagenesis are listed in Table 1.

Luciferase Reporter Assays. HepG2 and HuH7 cells were plated in 96-well plates and cultured overnight prior to transfection. Transfection was conducted in quadruplicate for each condition; each well was transfected with $100 \mathrm{ng}$ of each UTR luciferase reporter, $0.8 \mathrm{ng}$ pRL-null vector, and $30 \mathrm{nM}$ miRNA mimics of each miRNA or miR-neg as the negative control. Twenty-four hours after transfection, cells were lysed in passive lysis buffer and then subjected to the dualluciferase reporter assay according to the manufacturer's instructions (Promega). Firefly and Renilla luciferase activities were measured using a Packard TopCount luminescence and scintillation counter (PerkinElmer Life and Analytical Sciences, Waltham, MA). Firefly luciferase activity was first normalized to Renilla activity and the reporter activity was then presented relative to that of the control pGL3-promoter vector (set at a value of $100 \%$ ).

Western Blotting. HepG2 cells were transfected with miRNA mimics (miR-3664-3p or miR-neg) at $30 \mathrm{nM}$; 72 hours posttransfection, whole cell lysates were prepared with radioimmunoprecipitation assay buffer (50 mM Tris- $\mathrm{HCl}, \mathrm{pH} 7.4,150 \mathrm{mM} \mathrm{NaCl}, 1 \%$ NP-40, $2 \mathrm{mM}$ EDTA, 0.5\% sodium deoxycholate, and $0.1 \%$ sodium dodecyl sulfate). Protein concentrations of cell lysates were measured using the Bradford protein assay (Bio-Rad, Hercules, CA). Fifty micrograms of each cell lysate was subjected to SDS-PAGE on $12 \%$ acrylamide gels and transferred onto nitrocellulose membranes. The anti-UGT2B7 antibody used in Western blotting was developed in our laboratory as previously reported (Hu et al., 2014c). After the membranes were probed with primary antibodies (anti-UGT2B7 or 
TABLE 1

Primers used for cloning, site-directed mutagenesis, and RT-qPCR

\begin{tabular}{|c|c|}
\hline Primer & Sequence $\left(5^{\prime}-3^{\prime}\right)$ \\
\hline \multicolumn{2}{|l|}{ Cloning } \\
\hline UGT2B7_3'-UTR_F & CCGCTCTAGATTATATCTGAGATTTGAAGC \\
\hline UGT2B7_3'-UTR_R & CCGGTCTAGACCGTAGTGTTTTCTTCATTG \\
\hline UGT2B4_3'-UTR_F & CCGGTCTAGATTACGTCTGAGGCTGGAAGC \\
\hline UGT2B4_3'-UTR_R & CCGATCTAGAGCTTCCTCAACAACAGTTAA \\
\hline \multicolumn{2}{|l|}{ Site-directed mutagenesis } \\
\hline 2B7_miR3664_MT_F & AGATTTCTTTCTTAGCTAGACAAAAAAAAAAAAAG \\
\hline 2B7_miR3664_MT_R & TTTTTTTTTTTTGTCTAGCTAAGAAAGAAATCTTG \\
\hline 2B4_miR135a_MT_F & CAAAAATGATATAAATGACTATGAGGTTATATTG \\
\hline 2B4_miR135a_MT_R & CAATATAACCTCATAGTCATTTATATCATTTTTG \\
\hline 2B4_miR410_MT_F & AAAGCCATATGAGGTCCGCTTGAAATGTATTGAG \\
\hline 2B4_miR410_MT_R & CTCAATACATTTCAAGCGGACCTCATATGGCTTT \\
\hline \multicolumn{2}{|r|}{ 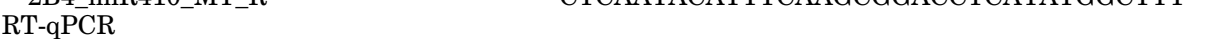 } \\
\hline miR3664-3p_qPCR F & GCAGTCTCAGGAGTAAAGACA \\
\hline miR3664-3p_qPCR_R & AGGTCCAGTTTTTTTTTTTTTTTAACTC \\
\hline miR-1266-5p_qPCR_F & GCC TCA GGG CTG TAG AAC A \\
\hline miR-1266-5p gPCR R & GTC CAG TTT TTT TTT TTT TTT AGC CC \\
\hline miR-4483_qPCR_F & GCA GGG GGT GGT CTG T \\
\hline miR-4483_qPCR_R & GGT CCA GTT TTT TTT TTT TTT TCA ACA \\
\hline miR-4317_qPCR_F & GCA GAC ATT GCC AGG GA \\
\hline miR-4317_qPCR_R & CAG GTC CAG TTT TTT TTT TTT TTT AAA C \\
\hline miR-216b-5p_qPCR_F & GCA GAA ATC TCT GCA GGC A \\
\hline miR-216b-5p_qPCR_R & GGT CCA GTT TTT TTT TTT TTT TTC ACA TT \\
\hline miR-135a_qPCR_F & CGC AGT ATG GCT TTT TAT TCC T \\
\hline miR-135a_qPCR_R & GGT CCA GTT TTT TTT TTT TTT TTC ACA T \\
\hline miR-410_qPCR_F & CGC AGA ATA TAA CAC AGA TGG C \\
\hline miR-410_qPCR_R & AGG TCC AGT TTT TTT TTT TTT TTA CAG \\
\hline miR-489 gPCR F & CGC AGG TGA CAT CAC ATA TAC \\
\hline miR-489_qPCR_R & CCA GTT TTT TTT TTT TTT TGC TGC C \\
\hline miR-4691-5p_qPCR_F & GGT CCT CCA GGC CAT GA \\
\hline miR-4691-5p_qPCR_R & AGT TTT TTT TTT TTT TTC CGC AGC \\
\hline miR-101_qPCR_F & CGC AGT ACA GTA CTG TGA TA \\
\hline miR-101_qPCR_R & GGT CCA GTT TTT TTT TTT TTT TTT CAG T \\
\hline
\end{tabular}

$\mathrm{F}$, forward; R, reverse.

anti-Calnexin [Sigma-Aldrich, St. Louis, MO]), the membranes were probed with horseradish peroxidase-conjugated donkey anti-rabbit secondary antibody (NeoMarkers, Fremont, CA). The SuperSignalWest Pico chemiluminescent kit (Thermo Fisher Scientific) and the LAS4000 luminescent image analyzer (GE Healthcare Life Sciences, Piscataway, NJ) were used for visualizing immunosignals. Band intensity was quantified using Multi Gauge version 3.0 image software (FUJIFILM, Tokyo, Japan).

Morphine Glucuronidation Assay. HepG2 cells were plated in six-well plates and cultured overnight before the transfection with mimics (miR-neg or miR-3664-3p) at $30 \mathrm{nM}$. Whole cell lysates were prepared after 24 hours post-transfection with $80 \mu \mathrm{l}$ Tris-HCl/EDTA buffer (10 mM Tris-HCl and $1 \mathrm{mM}$ EDTA, pH 7.6) and the protein concentrations of the lysates were determined as described above. A $200-\mu$ l reaction of each sample containing $100 \mathrm{mM}$ potassium phosphate, $\mathrm{pH} 7.4,4 \mathrm{mM} \mathrm{MgCl}_{2}, 5 \mathrm{mM}$ morphine, $125 \mu \mathrm{g}$ lysate protein, and $5 \mathrm{mM}$ UDPGA was incubated for 2 hours at $37^{\circ} \mathrm{C}$ in a shaking water bath. The glucuronidation reaction was terminated by the addition of $2 \mu \mathrm{l} 70 \%$ (v/v) perchloric acid and samples were kept on ice for 30 minutes before centrifugation at $5000 \mathrm{~g}$ for 10 minutes at $4^{\circ} \mathrm{C}$. The supernatant fraction (approximately $40 \mu \mathrm{l}$ ) was analyzed by highperformance liquid chromatography (HPLC) using an Agilent 1100 series instrument (Agilent Technologies, Sydney, NSW, Australia) as previously described (Uchaipichat et al., 2004). The HPLC column eluent was monitored by fluorescence detection at an excitation wavelength of $235 \mathrm{~nm}$ and an emission wavelength of $345 \mathrm{~nm}$. Quantification of morphine-3-glucuronide concentrations in the samples were calculated by comparing peak areas of the samples to those of standard curves prepared over the concentration ranges of 0.5 to $40 \mu \mathrm{M}$ morphine-3-glucuronide. Morphine-6-glucuronide concentration was not detected in the samples.

Data Analyses of Liver Hepatocellular Carcinoma. Transcriptome profiling data of RNA sequencing (RNAseq) and miRNA sequencing of liver hepatocellular carcinoma (LIHC) were downloaded from The Cancer Genome Atlas (TCGA) data portal (https://gdc-portal. nci.nih.gov/). The LIHC RNAseq expression data from 371 samples were represented in the form of high-throughput sequencing counts. Genes (protein coding and noncoding) with a mean of less than 10 counts were discarded; the counts of the remaining genes were normalized using the upper quantile normalization method. Correlation analyses between the expression levels of UGT and miRNA gene sets were conducted using the Spearman rank method and plots were drawn using the $\mathrm{R}$ statistical package (https://cran.r-project.org/; R Foundation for Statistical Computing, Vienna, Austria).

Statistical Analysis. Statistical analyses of all data were performed using GraphPad Prism 6 software (GraphPad Inc., La Jolla, $\mathrm{CA})$, with a two-tailed independent $t$ test. Correlation analyses between expression levels of a UGT gene and a miRNA in the panel of liver tissues or the panel of human tissues were conducted by Spearman correlation. A $P$ value of $<0.05$ was considered statistically significant.

\section{Results}

The 3'-UTRs of UGT2B7 and UGT2B4 Contain Putative miRNA Target Sites. Our sequence analysis of the UGT2B7 3'-UTR using TargetScan software (http://www.targetscan. org; Whitehead Institute for Biomedical Research, Cambridge, MA) identified putative target sites for five miRNAs: namely, miR-3664-3p, miR-1266-5p, miR-4483, miR-4317, and miR-216b-5p (Supplemental Fig. 1A). To test the potential functionalities of these sites, we cloned the UGT2B7 3'-UTR into the pGL3 vector downstream of the luciferase gene, generating the UGT2B7 3'-UTR luciferase reporter (termed 
pGL3/2B7/UTR). Cotransfection of pGL3/2B7/UTR with miRNA mimics targeting the five predicted binding sites in HepG2 cells showed that two of the five mimics (namely, miR-3664-3p and miR-216b-5p) significantly reduced the reporter activity by $51 \%$ and $22 \%$, respectively (Fig. $1 \mathrm{~A}$ ).

Our sequence analysis of the UGT2B4 3'-UTR using the TargetScan software predicted putative target sites for six microRNAs: namely, miR-135a-5p, miR-410-3p, miR-489-3p, miR-4691-5p, miR-216b-5p, and miR-101-3p (Supplemental Fig. 1B). We cloned the UGT2B4 3'-UTR into the pGL3 vector downstream of the luciferase gene, generating the UGT2B4 $3{ }^{\prime}$ UTR luciferase reporter (termed pGL3/2B4/UTR). Cotransfection of $\mathrm{pGL} 3 / 2 \mathrm{~B} 4 / \mathrm{UTR}$ with miRNA mimics targeting the six predicted binding sites in HepG2 cells showed that the reporter activity was significantly reduced by $39 \%, 30 \%, 22 \%$, $24 \%$, and $24 \%$ by miR-135a-5p, miR-410-3p, miR-489-3p, miR4691-5p, and miR-216b-5p, respectively (Fig. 1B). miR-101-3p mimics did not alter the reporter activity (Fig. 1B). The expression levels of the above-mentioned 10 miRNAs in HepG2 cells were measured by quantitative real-time PCR. As shown in Fig. 1C, miR-135a-5p and miR-101-3p were highly expressed; however, four miRNAs (miR-3664-3p, miR1266-5p, miR-4317, and miR-410-3p) were expressed at relatively low levels, and the remaining four miRNAs (miR-4483, miR-216b-5p, miR-489-3p, and miR-4691-5p) were not detectable. The downregulation of UGT2B4 and UGT2B7 by miR$216 \mathrm{~b}-5 \mathrm{p}$ in liver cancer cell lines was recently reported (Dluzen et al., 2016). In our study, we define the roles of miR-3664-3p, miR-135a-5p, and miR-410-3p in the regulation of UGT2B4 and $U G T 2 B 7$ in liver cancer cell lines.

Regulation of UGT2B7 by miR-3664-3p in Liver Cancer Cell Lines. The predicted miR-3664-3p binding site in the UGT2B7 3'-UTR is located at nucleotides 77-99 downstream of the UGT2B7 stop codon (Fig. 2A). This site is defined as a 8 mer site by TargetScan (pairing to the 2- to 7-nucleotide miRNA seed and nucleotide 8 plus an A at nucleotide 1) and also shows 3 '-sequence pairing of nucleotides $11-15$ to $\mathrm{miR}$ 3664-3p. A $3{ }^{\prime}$-sequence pairing at nucleotides 12-17 of miRNAs has been shown to enhance the efficacy of miRNA targeting (Grimson et al., 2007). To show a direct role of this site in the miR-3664-3p mimic-mediated reduction in pGL3/2B7/UTR reporter activity, the miR-3664-3p site in the pGL3/2B7/UTR reporter was mutated, generating the construct pGL3/2B7/UTR/miR3664/MT (Fig. 2B). miR-3664-3p mimics were cotransfected with wild-type pGL3/2B7/UTR or the pGL3/2B7/UTR/miR3664/MT reporter into HepG2 and HuH7 cells. The miR-3664-3p mimics significantly reduced the activity of the wild-type reporter in both cell lines; this reduction was abolished in HepG2 cells (Fig. 2C) or significantly abrogated in $\mathrm{HuH} 7$ cells (Fig. 2D) by a mutation of the miR-3664-3p site. These results indicate that $U G T 2 B 7$ is a direct target of miR-3664-3p in HepG2 and $\mathrm{HuH} 7$ cells.

The impact of miR-3664-3p on the expression and activity of UGT2B7 was tested in HepG2 cells using miRNA mimics and inhibitors. As expected, the miR-3664-3p mimics significantly reduced the UGT2B7 mRNA levels and the miR-3664-3p inhibitor significantly increased the UGT2B7 mRNA levels in HepG2 cells (Fig. 3A), while GAPDH mRNA levels were not significantly altered (Fig. 3B). Western blotting with a UGT2B7-specific antibody (Hu et al., 2014c) showed that UGT2B7 protein levels were reduced by $28 \%$ in HepG2 cells

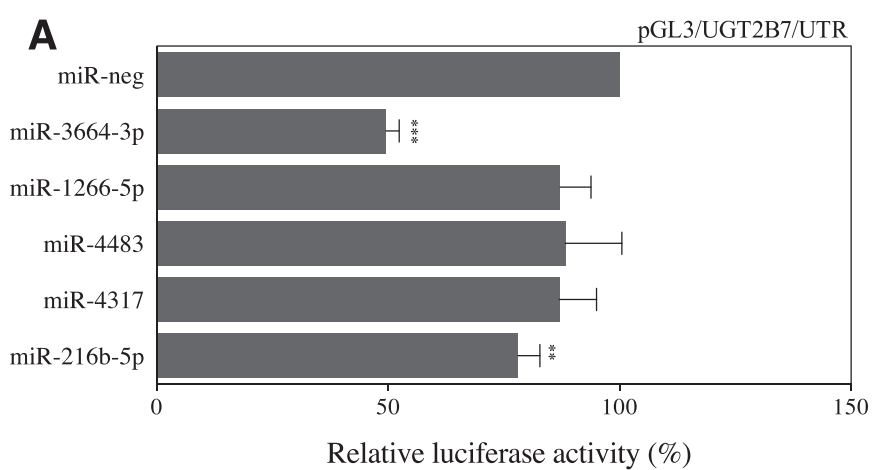

B

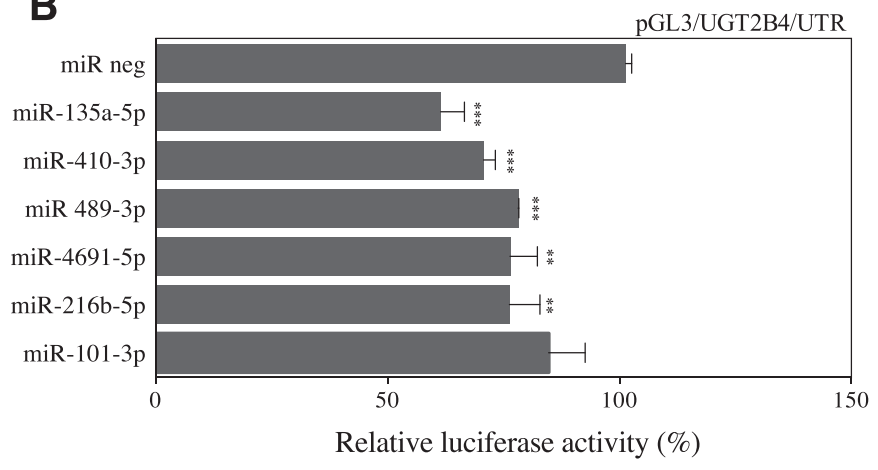

C

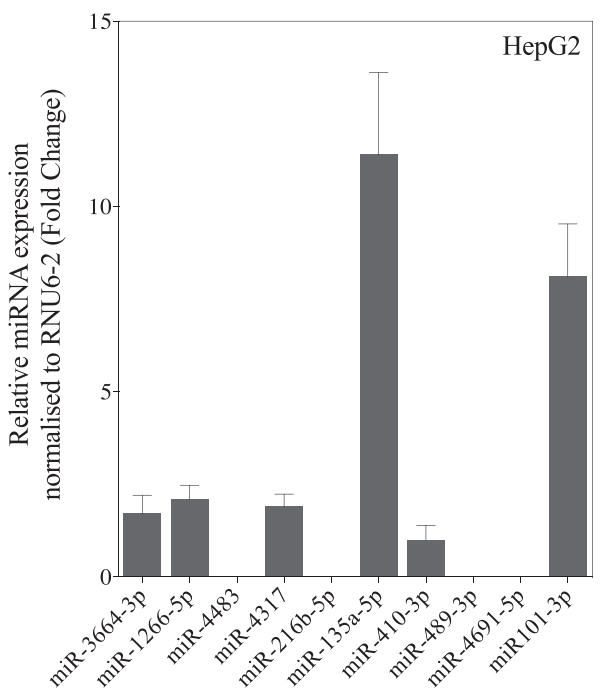

Fig. 1. Effects of miRNA mimics on a luciferase reporter containing the UGT2B7 3'-UTR or UGT2B4 3'-UTR and expression of miRNAs in HepG2 cells. (A and B) HepG2 cells were cotransfected with $30 \mathrm{nM}$ miR-neg or miRNA mimics targeting individual miRNAs, Renilla reporter vector as a control, and a luciferase reporter containing UGT2B7 3'-UTR (A) or UGT2B4 3'-UTR (B). Luciferase activity assays were performed as described in the Materials and Methods; the luciferase activity of the reporter cotransfected with miRNA mimics is presented relative to that of the reporter cotransfected with miR-neg (set as a value of $100 \%$ ). Data shown are means \pm S.E.M from four (A) or two (B) independent experiments performed in quadruplicate. ${ }^{*} P<0.005$; $* * * P<0.0005$. (C) The expression levels of miRNAs in HepG2 cells were measured using RT-qPCR, normalized toRNU6-2, and then presented relative to that of miR-410-3p (set at a value of 1 ). Data shown are means \pm S.E.M from a single experiment in triplicate.

transfected with miR-3664-3p mimics relative to the miR-neg control (Fig. 3C). UGT2B7 glucuronidation activity was tested by HPLC assay using morphine as a substrate. There was a significant $41 \%$ reduction in morphine glucuronidation in 


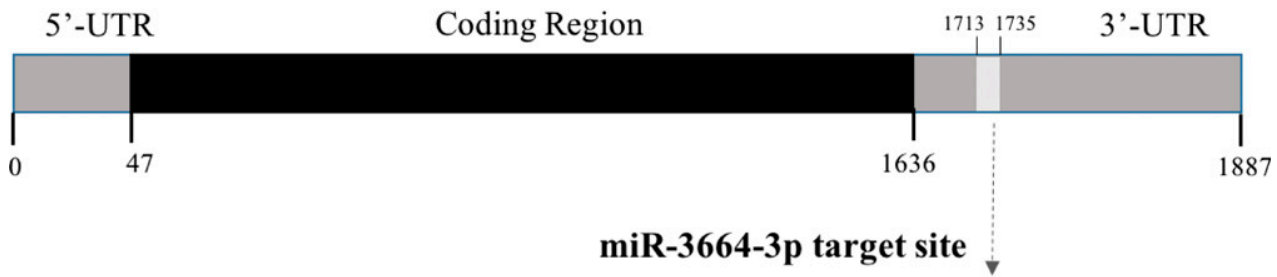

\begin{tabular}{lll}
\hline Position 77-99 of & & \\
UGT2B7 3' UTR & $5^{\prime}$ & GCAAGAUUUCUUUCUUCCUGAGA \\
hsa-miR-3664-3p & $3^{\prime}$ & UUGAGACAGAAAUGAGGACUCU
\end{tabular}

B

\begin{tabular}{|c|c|c|}
\hline \multirow[b]{2}{*}{ pGL3/2B7/UTR (wild type) } & \multirow[b]{2}{*}{ Firefly Luciferase } & 3'UTR of $U G T 2 B 7$ \\
\hline & & TTTCTTTCTTССТGAGACAAAAAA \\
\hline & & 3'UTR of $U G T 2 B 7$ \\
\hline pGL3/2B7/UTR/miR-3664/MT (mutant) & Firefly Luciferase & TTTCTTTCTTAAGCTAGACAAAAAA \\
\hline
\end{tabular}

C

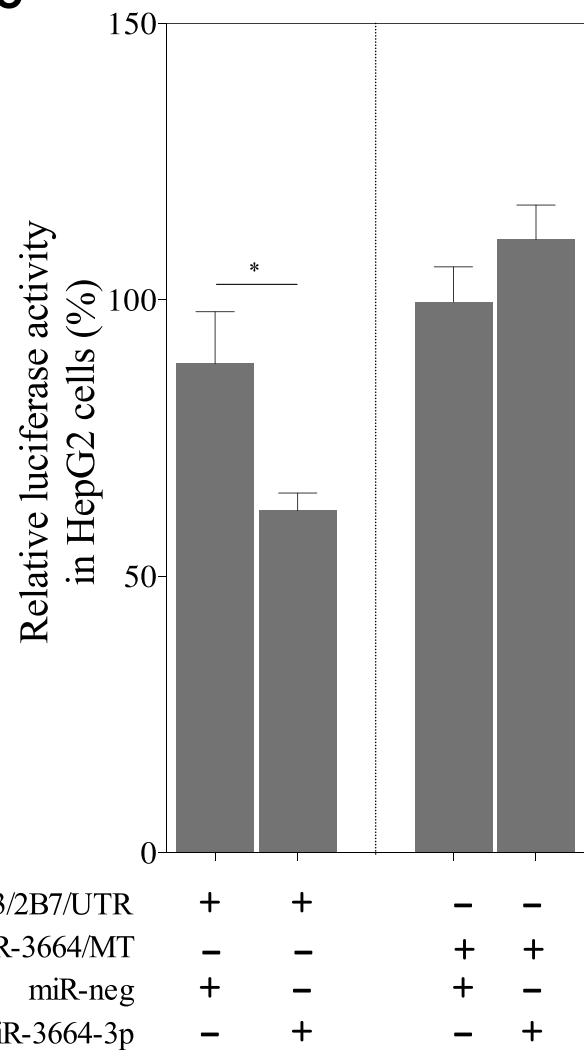

D

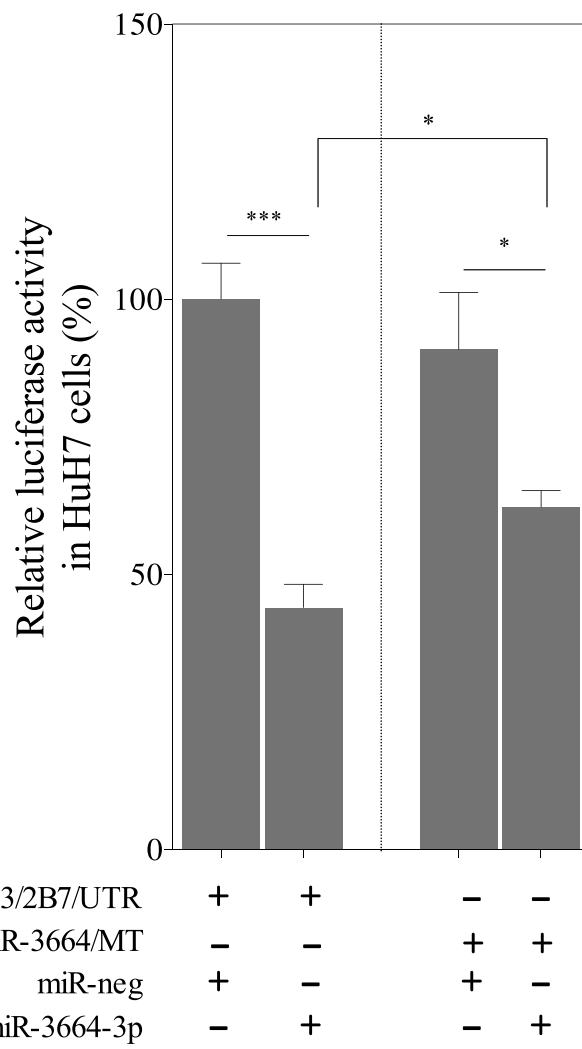

Fig. 2. A functional miR-3664-3p target site in the UGT2B7 3'-UTR. (A) Shown are the position of a miR-3664-3p target site in the UGT2B7 3'-UTR and the sequence complementarity between this site and miR-3664-3p, including seed pairing (highlighted in gray) and substantial $3{ }^{\prime}$-sequence pairing. The nucleotides of the miR-3664-3p sites are numbered relative to the stop codon (TAG with G positioned as -1) (NM_001074.3). (B) The mutation of CCTC to AGCT (boxed) that abolished the seed pairing between the miR-3664-3p target site and miR-3664-3p is shown. (C and D) Cotransfection of the UGT2B7 3'-UTR luciferase reporter carrying either the wild-type or mutated miR-3664-3p site, $30 \mathrm{nM}$ miR-neg or miR-3664-3p mimics, and Renilla reporter vector as the control in HepG2 (C) and HuH7 (D) cells; 24 hours post-transfection, cells were harvested for luciferase activity assays as described in the Materials and Methods. The luciferase activity of the reporter cotransfected with miR-3664-3p is presented relative to that of the reporter cotransfected with miR-neg (set as a value of $100 \%$ ). Data shown are means \pm S.E.M from three (C) or two (D) independent experiments performed in quadruplicate. $* P<0.01 ; * * P<0.005 ; * * * P<0.0005$. 


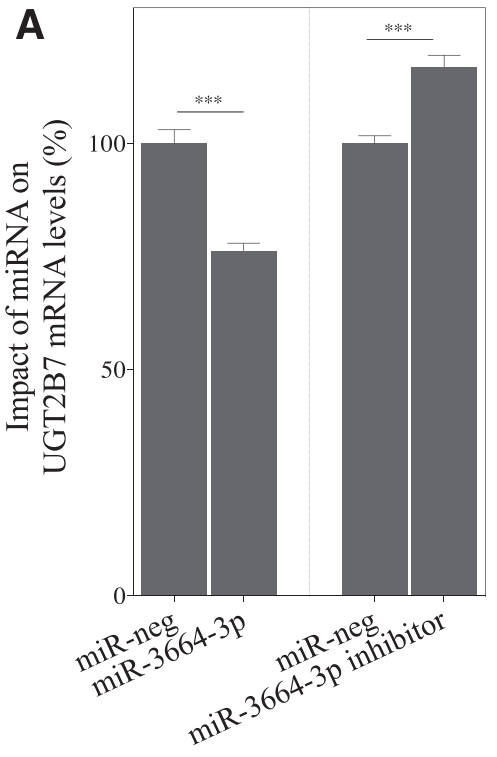

C $\longleftarrow$ miR-neg $\rightarrow \leftarrow$ miR-3664 $\rightarrow$

$==$ UGT2B7; 50kDa = $\simeq$ C. Calnexin; 90kDa
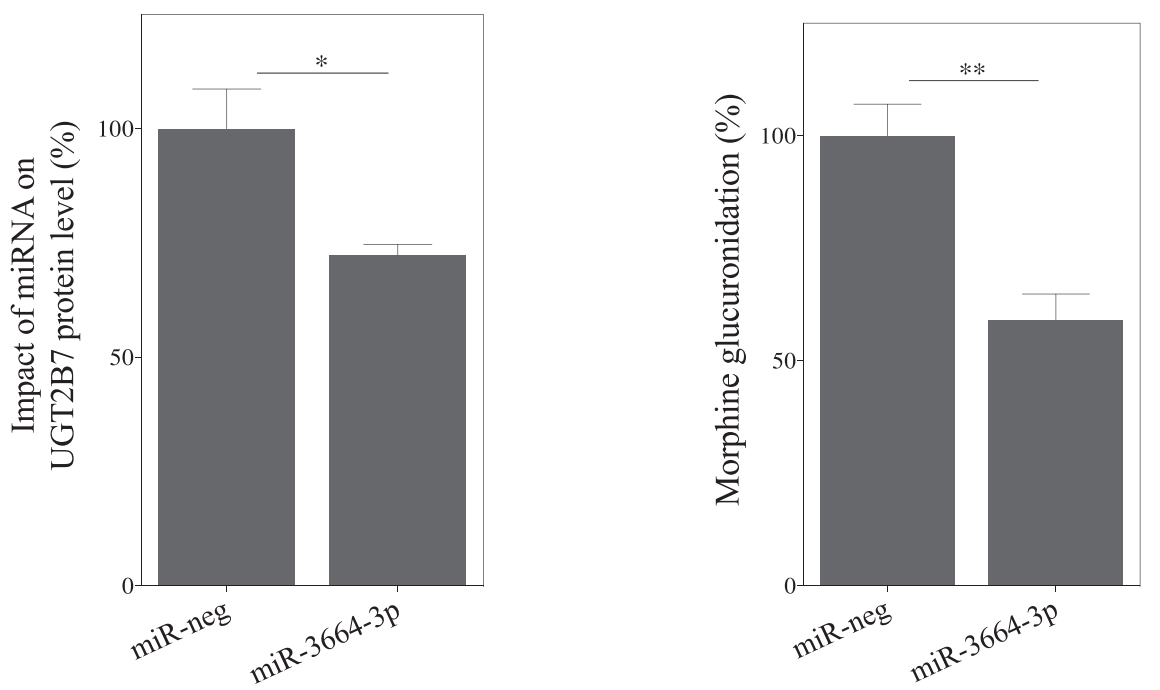
mir-nes $m i R-3664-3 p$

E

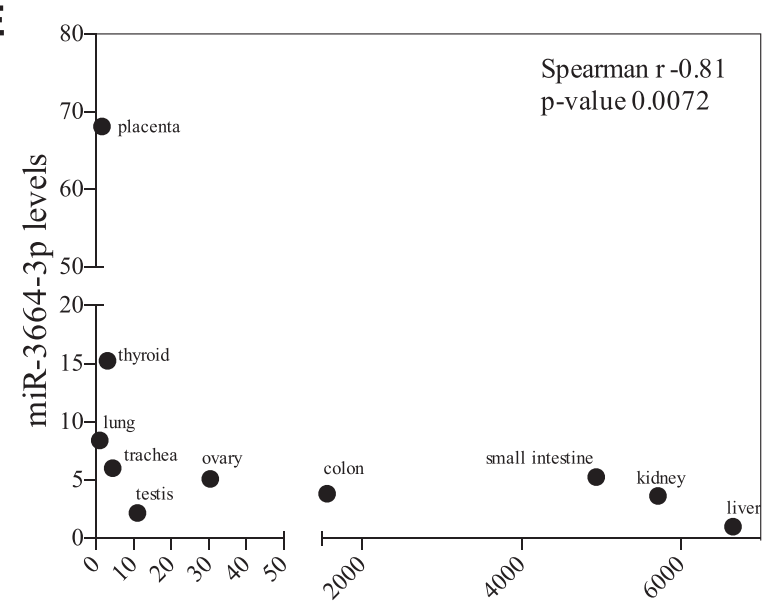

UGT2B7 mRNA levels
Fig. 3. miR-3664-3p mimics reduce the expression and activity of endogenous UGT2B7, and miR-3664 levels are negatively correlated to UGT2B7 mRNA in a tissue RNA panel. HepG2 cells were transfected with miR-3664-3p mimics (or miR-3664-3p inhibitor) or miR-neg. (A, C, and D) Cells were harvested for 1) analysis of UGT2B7 mRNA levels by RT-qPCR (A), 2) analysis of UGT2B7 protein levels by Western blotting (C), and 3) analysis of UGT2B7 activity via morphine glucuronidation assays $\mathrm{D}$ ) as described in the Materials and Methods. (A and B) UGT2B7 and GAPDH mRNA levels in cells transfected with miR-3664-3p mimics (or miR-3664-3p inhibitors) are presented relative to that in cells transfected the negative control miR-neg. Data shown are means \pm S.E.M. from three independent experiments performed in triplicate. $* * * P<0.0005$. (C) UGT2B7 and calnexin protein immunosignals from a representative Western blot experiment (top); quantification of UGT2B7 protein levels (means \pm S.E.M. from two independent experiments performed in triplicate) in cells transfected miR-3664-3p mimics relative to cells transfected with miR-neg (bottom). $* P<0.05$. (D) Morphine-glucuronidation activity in miR3664-3p mimic-transfected cells relative to that of miR-neg-transfected cells (set as a value of $100 \%)$. Data shown are means \pm S.E.M. from two independent experiments performed in triplicate. $* * P<0.005$. (E) miR-3664 and UGT2B4 mRNA levels in a panel of 10 normal tissues were measured using RT-qPCR as described in the Materials and Methods. Shown is a Spearman correlation analysis between the expression levels of miR-3664-3p and UGT2B4 mRNA in this human tissue panel. 
HepG2 cells transfected with miR-3664-3p mimics relative to the miR-neg control (Fig. 3D). Collectively, these data define a novel miR-3664-3p target site in the UGT2B7 3'-UTR that negatively regulates UGT2B7 expression and activity in liver cancer cell lines.

To assess whether miR-3664-3p might play a role in the expression of UGT2B7 in the normal liver, we measured the expression levels of miR-3664-3p and UGT2B7 in a panel of 18 normal human liver tissues using RT-qPCR. Consistent with previous reports (Congiu et al., 2002; Court, 2010), UGT2B7 mRNA levels were very high in liver tissues. In contrast, levels of miR-3664-3p were extremely low or undetectable (data not shown). An analysis of RNAseq data from a TCGA cohort of 371 LIHCs also revealed high levels of UGT2B7 mRNA and extremely low levels of miR-3664 (data not shown). These observations suggest a negative correlation between miR-3664 and UGT2B7 mRNA levels in both normal and cancerous liver tissues. Although an overall correlation analysis between miR-3664 and UGT2B7 mRNA levels in liver cancer/liver tissue was not possible due to the lack of miR-3664 expression in most samples, we did measure a significant negative correlation between the expression levels of UGT2B7 and miR-3664-3p in a panel of 10 normal human tissues that did express both UGT2B7 and miR-3664-3p (liver, kidney, colon, small intestine, ovary, testis, trachea, lung, thyroid, and placenta) (Pearson $r=-0.081, P<0.01$ ) (Fig. 3E).

Regulation of UGT2B4 by miR-135a-5p and miR-410-3p in Liver Cancer Cell Lines. The predicted miR-135a-5p binding site in the UGT2B4 $3^{\prime}$-UTR is located at nucleotides 202-224 downstream of the stop codon and is defined as an 8 mer site by TargetScan (Fig. 2B). In addition to the seed pairing, the miR-135a-5p binding site has 3 '-sequence pairing to miR-135a-5p (Fig. 4A). The predicted miR-410-3p binding site is located at nucleotides 213-235 downstream of the UGT2B4 stop codon and is defined as a 7mer-m8 site (pairing to the 2- to 7-nucleotide miRNA seed and nucleotide 8 ). The miR-410-3p binding site also has substantial 3 '-sequence pairing to miR-410-3p including nucleotides 13-17 (Fig. 4A). Of note, the $5^{\prime} 11$ nucleotides (5'AUAAAGCCAUA3') of the miR-135a-5p target site overlap with the $3^{\prime} 11$ nucleotides of the miR-410-5p target site in the UGT2B4 3'-UTR (Supplemental Fig. 1B). To investigate the regulation of UGT2B4 by miR135a-5p and miR-410-3p through these two predicted sites, we mutated the sites in the pGL3/2B4/UTR reporter construct to generate the pGL3/2B4/UTR/miR135a/MT and pGL3/2B4/UTR/ miR410/MT constructs (Fig. 4B). Cotransfection of miR-135a-5p mimics with the reporter constructs showed that miR-135a-5p significantly decreased the activity of the wild-type reporter but did not significantly alter the activity of pGL3/2B4/UTR/ miR135a/MT in both HepG2 (Fig. 4C) and HuH7 (Fig. 4D) cells. Cotransfection of miR-410-3p mimics with the reporters showed that miR-410-3p significantly reduced the activity of the wild-type reporter but not that of pGL3/2B4/UTR/ miR410/MT in HepG2 cells (Fig. 4D).

To assess these miRNAs in the regulation of endogenous UGT2B4 mRNA levels, we transfected miRNA mimics targeting miR-135a-5p or miR-410-3p in HuH7 cells; these cells were used because they had higher levels of UGT2B4 expression relative to HepG2 cells (see Supplemental Fig. 2). Both miR-135a-5p and miR-410-3p mimics significantly reduced UGT2B4 mRNA levels (Fig. 5A) but did not change GAPDH mRNA levels (Fig. 5B). Collectively, these results indicate that
UGT2B4 is a direct target of miR-135a-5p and miR-410-3p in liver cancer cells. However, the potential impact of these miRNAs on UGT2B4 protein level or enzymatic activity could not be assessed due to the lack of a UGT2B4-specific antibody or a probe substrate that would be specific for UGT2B4 in HepG2 or HuH7 cells.

The relationship between miR-135a-5p and UGT2B4 expression levels was assessed in a panel of 18 normal human liver tissues using quantitative real-time PCR, revealing a significant negative correlation (Spearman $r=-0.47, P=$ 0.047) (Fig. 5C). We also measured miR-135a-5p and UGT2B4 levels in HepG2 and Huh7 cells and found that miR-135a-5p levels are significantly lower in Huh7 cells than in HepG2 cells; conversely, UGT2B4 levels are significantly higher in Huh7 cells than in HepG2 cells (Supplemental Fig. 2), suggesting that negative correlation extends to these liver cancer cell lines. In contrast, when we examined miR-135a and UGT2B4 levels in the TCGA-LIHC RNAseq dataset containing 371 hepatocellular carcinoma specimens, we saw no correlation (Supplemental Fig. 3). Finally, we measured miR-135a-5p and UGT2B4 levels in a panel of 10 normal human tissues (liver, kidney, colon, small intestine, ovary, testis, trachea, lung, thyroid, and placenta). Both genes varied widely in expression but were uncorrelated (data not shown).

No correlation was observed between UGT2B4 and miR410-3p expression levels in the normal human liver tissue panel (data not shown). However, analysis of the TCGA-LIHC RNAseq dataset (371 hepatocellular carcinoma specimens) revealed a significant negative correlation between miR-410 and UGT2B4 mRNA levels (Spearman $r=-0.15, P=0.002$ ) (Fig. 5D). Furthermore, we also observed a significant negative correlation between the expression levels of UGT2B4 and miR-410-3p in the panel of 10 normal human tissues (liver, kidney, testis, lung, thyroid, placenta, prostate, heart, and cervix) (Pearson $r=-0.76, P=0.010$ ) (Fig. 5E).

\section{Discussion}

UGT2B4 and UGT2B7 are highly expressed in the liver. While their transcriptional regulation has been well studied (Hu et al., 2014b), to date there has been only one report of their post-transcriptional regulation by miR-216b-5p in liver cancer cell lines (Dluzen et al., 2016). We used bioinformatics analysis to predict multiple potential miRNA binding sites in the UGT2B7 and UGT2B4 3'-UTRs, and we were able to confirm a subset of these experimentally. In particular, we used reporter analysis, mutagenesis, and endogenous gene expression analysis to show that miR-3664-3p negatively regulates the UGT2B7 mRNA via a binding site in the $3^{\prime}$ UTR. We also showed inhibition of UGT2B7 at both protein and activity levels using a specific antibody and probe substrate. Similarly, we found that miR-135a-5p and miR-410-3p bind and regulate the UGT2B4 mRNA. Both miR-410 (Wang et al., 2014; Marrone et al., 2016) and miR-135a (Liu et al., 2012; Zeng et al., 2016) are reported to be upregulated in liver cancer and are considered as liver onco-miRs. By contrast, these two miRNAs are frequently downregulated in some other cancers, including breast, prostate, and pancreatic cancer (Chien et al., 2011; Gattolliat et al., 2011; Tang et al., 2014; Theodore et al., 2014). Overexpression of miR-3664 in the peripheral blood of patients with glioblastoma (Dong et al., 2014) and individuals with gastric cancer (Liu et al., 2014) was 

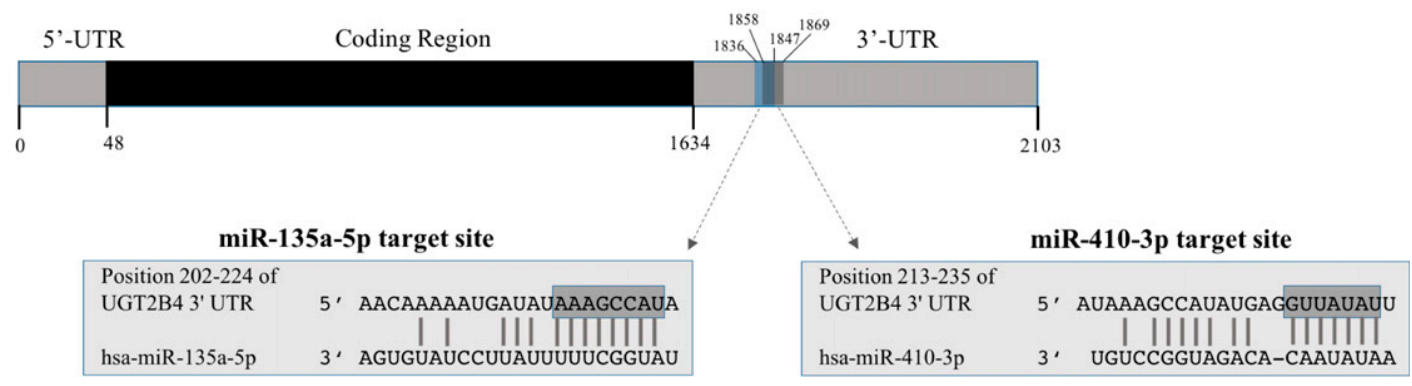

B
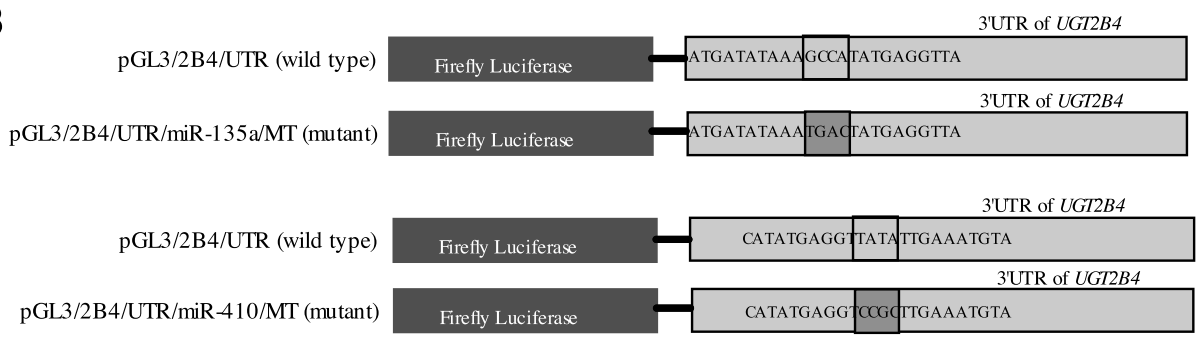

C

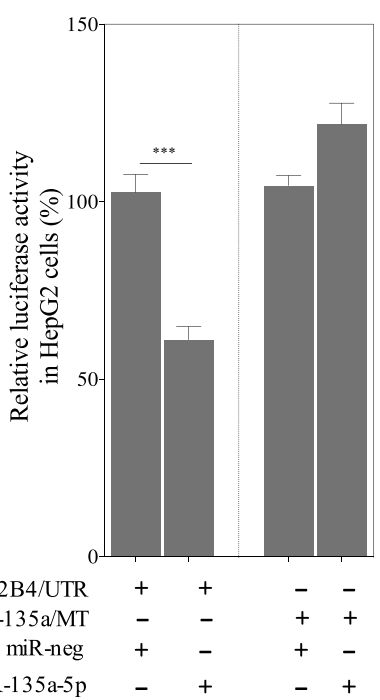

D

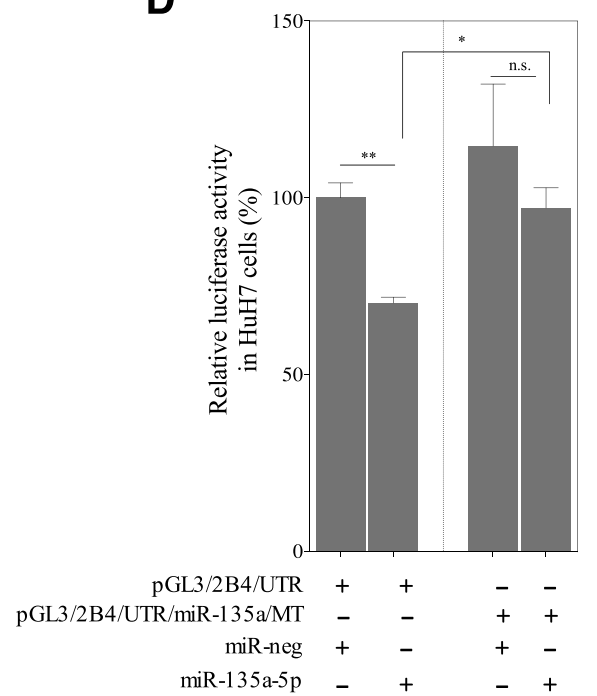

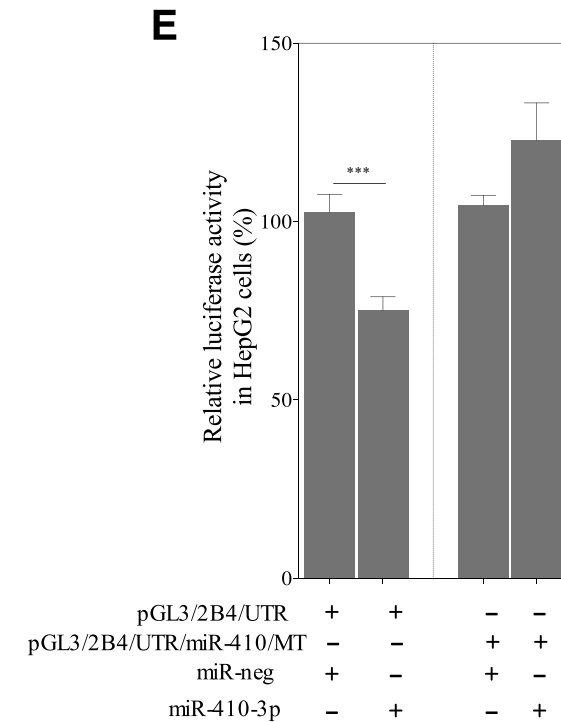

E

Fig. 4. A functional miR-135a-5p site and a functional miR-410-3p in the UGT2B4 3'-UTR. (A) Shown are the positions of the miR-135a-5p and the miR410-3p target site in the UGT2B4 3'-UTR and the sequence complementarity between these two sites and their cognate miRNAs, including seed pairing (highlighted in gray) and 3'-sequence pairing. The nucleotides of the two miRNA sites are numbered relative to the stop codon (TAG with G positioned as -1) (NM_021139.2). (B) The mutation of GCCA to TGAC (boxed) that abolishes the seed pairing between the miR-135a-5p target site and miR-135a$5 \mathrm{p}$, and the mutation of TATA to CCGC (boxed) that abolishes the seed pairing between the miR-410-3p target site and miR-410-3p is shown. (C and D) Cotransfection of a UGT2B4 3'-UTR luciferase reporter carrying either a wild-type or mutated miR-135a-5p target site, $30 \mathrm{nM}$ miR-neg or miR-135a-5p mimics, and Renilla reporter vector as the control in HepG2 (C) and HuH7 (D) cells. (E) Cotransfection of a UGT2B4 3'-UTR luciferase reporter carrying either a wild-type or mutated miR-410-3p target site, $30 \mathrm{nM}$ miR-neg or miR-410-3p mimics, and Renilla reporter vector as control in HepG2 cells. Twenty-four hours post-transfection, cells were harvested for luciferase activity assays as described in the Materials and Methods. The luciferase activity of the reporter cotransfected with miRNA mimics is presented relative to that of the reporter cotransfected with miR-neg (set as a value of 100\%). Data shown are means \pm S.E.M from two independent experiments performed in quadruplicate. ${ }^{*} P<0.05$; ${ }^{* *} P<0.005$; $* * * P<0.0005$. n.s., not significant.

recently reported; however, the biologic role of miR-3664-3p in liver cancer remains to be investigated.

miRNAs generally repress gene expression through mRNA degradation or translational repression depending on the degree and nature of sequence complementarity between the miRNA guide and mRNA target (Macfarlane and Murphy, 2010). Extensive base-pairing between the miRNA guide and mRNA target permits mRNA cleavage by Argonaute (Ago2) (Macfarlane and Murphy, 2010); expression levels of such
miRNAs and their targets may thus be negatively correlated due to miRNA-mediated mRNA degradation. The three functional miR-binding sites (UGT2B4 miR-135a-5p, UGT2B4 miR-410-3p, and UGT2B7 3664-3p) reported in our study showed (Figs. 2A and 4A) extensive seed pairing and additional 3 '-sequence pairing(s) with their targets, which would be expected to favor the mRNA degradation pathway.

Given the predicted role of each of the miRNAs that we studied in target mRNA degradation, we further predicted 
A

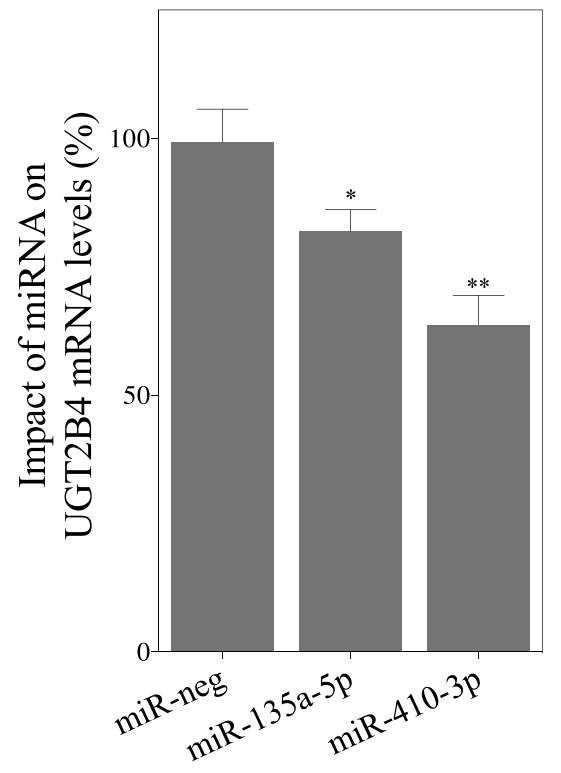

C

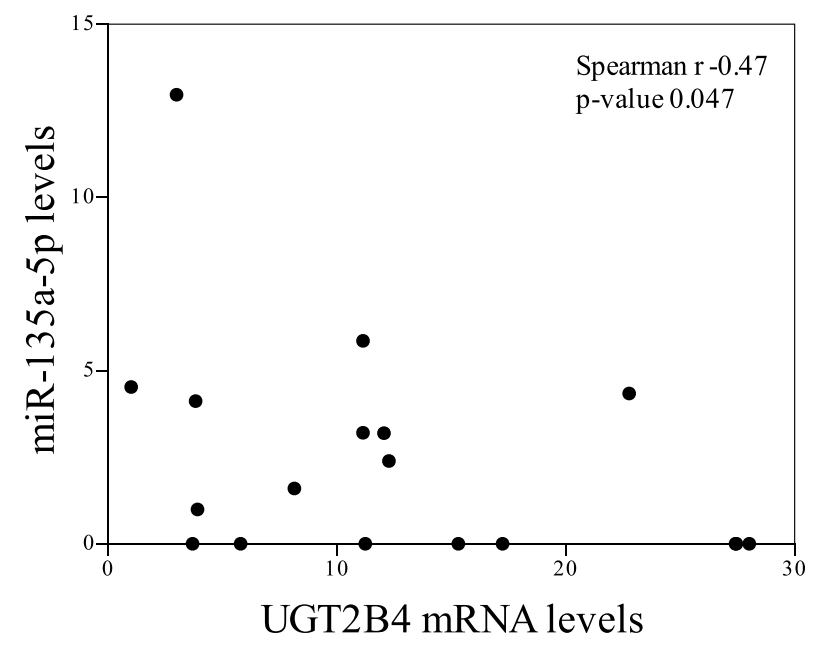

B

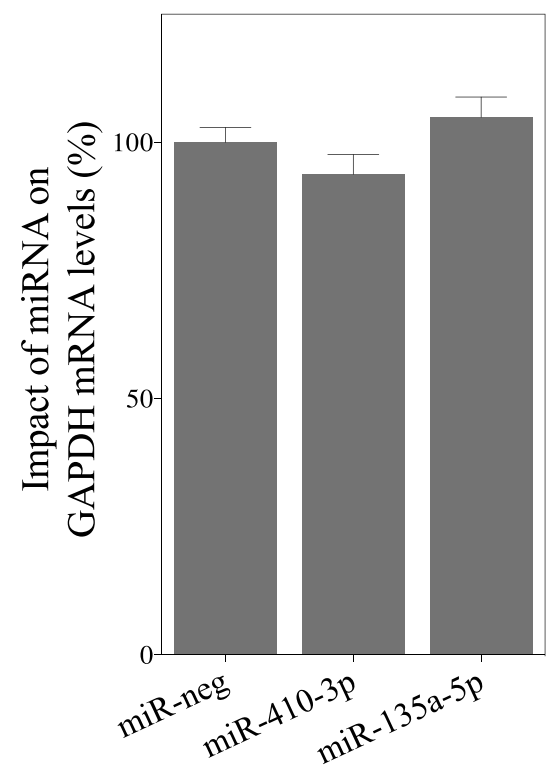

D

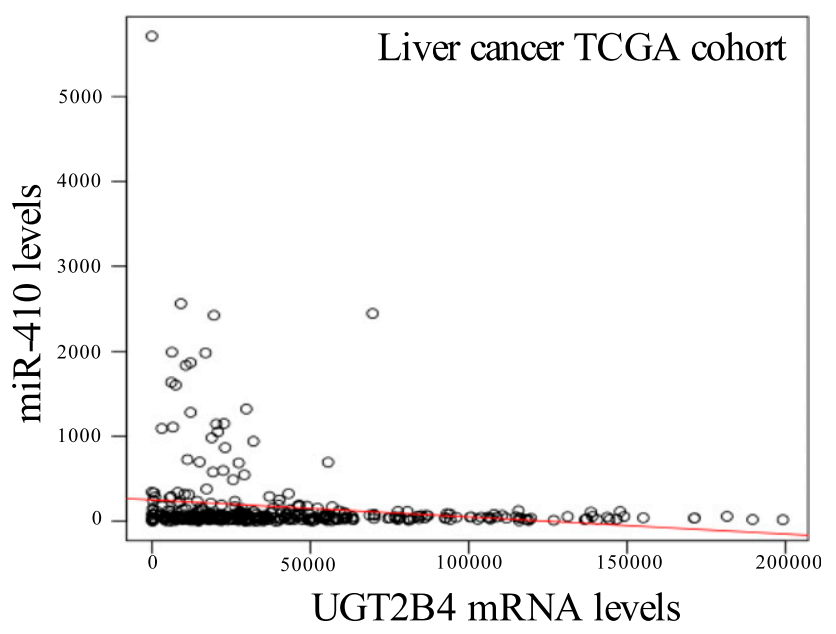

E

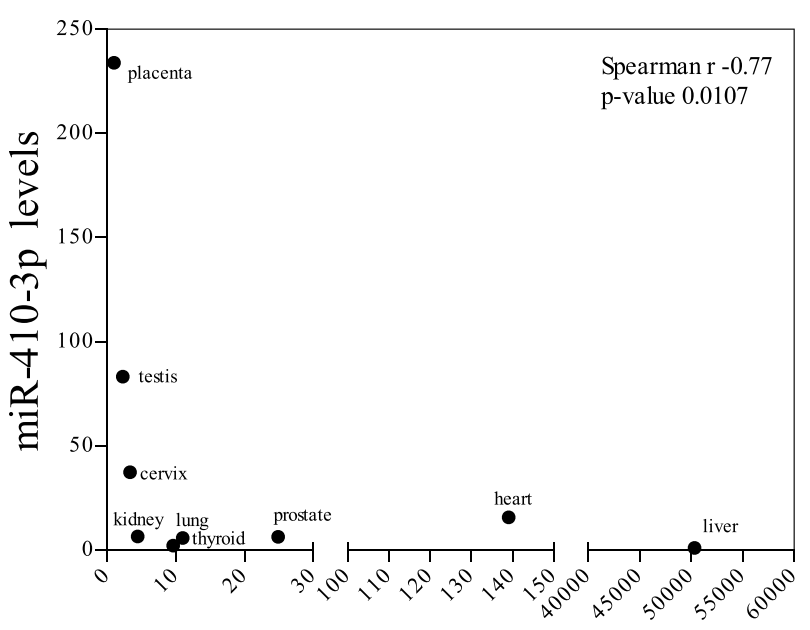

UGT2B4 mRNA levels

Fig. 5. miR-135a-5p and miR-410-3p mimics reduce UGT2B4 mRNA levels in HuH7 cells, and correlation analyses between UGT2B4 and miR-135a-5p (or miR-410-3p) levels in human tissue panels and liver cancer specimens are presented. (A and B) HuH7 cells were transfected with miR-neg, miR-135a$5 \mathrm{p}$, or miR-410-3p mimics. UGT2B4 and GAPDH mRNA levels were measured using RT-qPCR. The UGT2B4 mRNA levels in miRNA mimic-transfected cells are presented relative to that of cells transfected with miR-neg (set at a value of $100 \%$ ). Data shown are means \pm S.E.M. from two independent 
that negative correlations might be observed between miRNA levels and target mRNA levels in various tissues. Although this was the case for some of our miRNA-mRNA pairings, it was not necessarily seen in all of the contexts examined. Moreover when interpreting our data, it is important to consider that correlation alone does not imply causation, and many regulatory factors, both transcriptional and posttranscriptional, are involved in the regulation of a given gene. In the case of miR-3664 and UGT2B7, levels were negatively correlated in both normal and cancerous liver tissues with high expression of UGT2B7 and low (or absent) expression of miR-3664. One reasonable interpretation of this result is that the absence (or very low level) of miR-3664 permits a high steady-state level of UGT2B7 mRNA to be maintained. However, this causal relationship would need to be explicitly tested to state definitively that miR-3664-3p is an important endogenous regulator of basal UGT2B7 levels. Interestingly, in the analysis of the 10-tissue panel (including nine extrahepatic tissues), while UGT2B7 and miR-3664-3p levels were still negatively correlated overall, there were some tissues with low or absent UGT2B7 levels (ovary, trachea, and testis) that also had very low miR-3664-3p levels. This may be explained by considering the role of transcriptional (promoter level) regulation in determining the overall level of UGT2B7 expression. The key positive transcriptional regulators that activate the UGT2B7 promoter in the liver (e.g., HNFs) have little activity in tissues such as the trachea, ovary, and testis; hence, we expect low or absent UGT2B7 transcription. In this context, post-transcriptional regulation may have little relevance.

We found a negative correlation between miR-410 and UGT2B4 mRNA levels in the liver cancer TCGA cohort of 371 specimens as well as in the panel of 10 normal human tissues, but unexpectedly, not in the panel of 18 normal livers. This discrepancy remains to be resolved and may be clarified in the future by examining larger cohorts of normal livers and liver cancers.

The relationship between miR-135a and UGT2B4 mRNA levels in the various samples that we analyzed was also complex. Although there was a negative correlation in the panel of 18 normal livers, there was no correlation between the levels of these genes in the liver cancer TCGA cohort. This could be related to deregulation of other miRNAs and transcription factors that are involved in controlling UGT2B4 expression in liver cancer (Negrini et al., 2011; Callegari et al., 2015). Of note, Dluzen et al. (2014) reported the regulation of three $U G T 1 A$ genes $(1 \mathrm{~A} 1,1 \mathrm{~A} 3,1 \mathrm{~A} 6)$ by miR-491-3p in liver cancer cells and a significant inverse correlation between miR491-3p and mRNA levels of UGT1A3 and UGT1A6 (but not UGT1A1) in a panel of normal human liver specimens.

Several studies have investigated the cooperative regulation of single-target mRNAs by multiple miRNAs and revealed that the efficacy and cooperativity between miRNA target sites is predominantly determined by the interval spacing between seed sites (Doench and Sharp, 2004; Grimson et al., 2007; Saetrom et al., 2007; Pasquinelli, 2012). Specifically, the most effective cooperative downregulation is seen when the distance of two seed sites is between 13 and 35 nucleotides; however, this cooperativity becomes less likely when the internal spacing between miRNA seed sites is over 70 nucleotides (Saetrom et al., 2007). The seed site is defined as nucleotides 2-8 from the 3 ' end of a miRNA target site that are normally complementary to the seed sequence of the cognate miRNA (Bartel, 2009). Our findings, together with the recent report by Dluzen et al. (2016), indicate that UGT2B4 is a direct target of three miRNAs (miR-135a-5p, miR-410-3p, and miR$216 \mathrm{~b}-5 \mathrm{p}$ ). As mentioned earlier, the $5^{\prime} 11$ nucleotides of the miR-135a-5p site overlap with the $3^{\prime} 11$ nucleotides of the miR-410-3p site in the UGT2B4 3'-UTR (Supplemental Fig. 1B). The seed sites of these two miRNAs are separated by 12 nucleotides, which falls just outside of the proposed optimal spacing range; whether miR-135a-5p and miR-410-3p could cooperatively regulate UGT2B4 remains to be investigated in future studies. The miR-135a-5p site (or miR-410-3p site) and the miR-216b-5p site are separated by 137 nucleotides (or 149 nucleotides) in the UGT2B4 3'-UTR (Supplemental Fig. $1 \mathrm{~B}$ ), and the miR-3664-3p site and the recently reported miR216-5p site (Dluzen et al., 2016) are 73 nucleotides apart in the UGT2B7 3'-UTR (Supplemental Fig. 1A). Therefore, the cooperativity between these miRNA target sites is predicted to be less likely; however, this also remains to be tested.

When we initially screened the predicted miRNA binding sites using luciferase reporters, we also found repression of the UGT2B4 3'-UTR reporter by miR-4691-5p and miR-489-3p (Fig. 1B). The putative binding sites for miR-4691-5p and miR-489-3p in the UGT2B4 3'-UTR include overlapping seed sequences (Supplemental Fig. 1B). Thus, miR-4691-5p and miR-489-3p could possibly inhibit each other's activity based on a model of competitive loading of miRNA-activated miRNAinduced silencing complexes (Macfarlane and Murphy, 2010), In contrast, cooperative regulation between the miR-135a-3p and miR-4691-5p (or miR-489-3p) target sites may be possible, as the spacing between their seed sites is within the optimal range (13-35 nucleotides) for cooperativity. Again, future studies are required to test these possibilities.

In summary, this study demonstrates that UGT2B4 is a direct target of miR-135a-5p and miR-410-3p and that UGT2B7 is a direct target of miR-3664-3p in liver cancer cells. Combined with other recent reports of miRNA-mediated UGT regulation (Dluzen et al., 2014, 2016; Wijayakumara et al., 2015; Margaillan et al., 2016), our observations suggest that post-transcriptional regulation of UGTs by miRNAs may have an important role in fine-tuning glucuronidation activity in the liver as well as extrahepatic tissues. Although none of the miRNAs characterized in this study are predicted to have a role in cancer-specific UGT regulation, further analysis of the functional significance of the miRNA-regulatory network is ongoing. In particular, it is interesting to consider whether

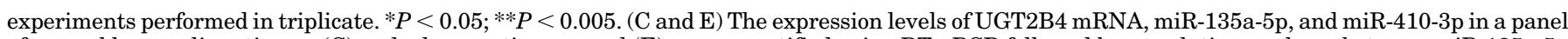

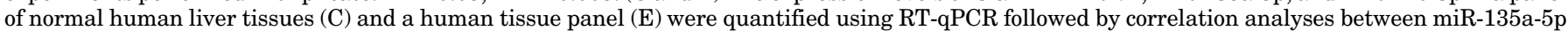

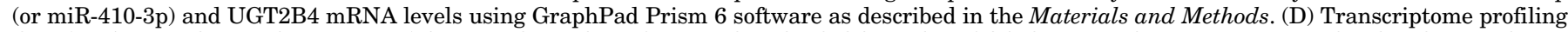

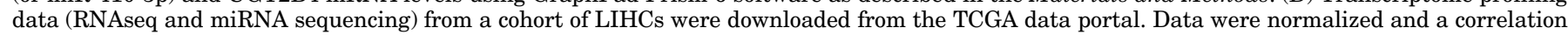

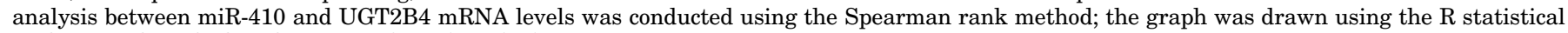
package as described in the Materials and Methods.
} 
deregulation of miRNA expression due to cellular stress or inflammation could alter UGT levels and hence the capacity for detoxification, which in turn may affect cancer risk.

\section{Authorship Contributions}

Participated in research design: Wijayakumara, Mackenzie, Hu, Meech.

Conducted experiments: Wijayakumara.

Performed data analysis: Wijayakumara, Mackenzie, Hu, Meech.

Wrote or contributed to the writing of the manuscript: Wijayakumara, Mackenzie, McKinnon, Hu, Meech.

\section{References}

Argikar UA and Remmel RP (2009) Effect of aging on glucuronidation of valproic acid in human liver microsomes and the role of UDP-glucuronosyltransferase UGT1A4, UGT1A8, and UGT1A10. Drug Metab Dispos 37.229-236.

Balcells I, Cirera S, and Busk PK (2011) Specific and sensitive quantitative RT-PCR of miRNAs with DNA primers. BMC Biotechnol 11:70.

Barbier O, Duran-Sandoval D, Pineda-Torra I, Kosykh V, Fruchart JC, and Staels B (2003a) Peroxisome proliferator-activated receptor alpha induces hepatic expression of the human bile acid glucuronidating UDP-glucuronosyltransferase 2B4 enzyme. J Biol Chem 278:32852-32860.

Barbier O, Torra IP, Sirvent A, Claudel T, Blanquart C, Duran-Sandoval D, Kuipers F, Kosykh V, Fruchart JC, and Staels B (2003b) FXR induces the UGT2B4 enzyme in hepatocytes: a potential mechanism of negative feedback control of FXR activity. Gastroenterology 124:1926-1940.

Barre L, Fournel-Gigleux S, Finel M, Netter P, Magdalou J, and Ouzzine M (2007) Substrate specificity of the human UDP-glucuronosyltransferase UGT2B4 and UGT2B7. Identification of a critical aromatic amino acid residue at position 33 FEBS $J$ 274:1256-1264.

Bartel DP (2009) MicroRNAs: target recognition and regulatory functions. Cell 136 215-233

Callegari E, Gramantieri L, Domenicali M, D'Abundo L, Sabbioni S, and Negrini M (2015) MicroRNAs in liver cancer: a model for investigating pathogenesis and novel therapeutic approaches. Cell Death Differ 22:46-57.

Chien WW, Domenech C, Catallo R, Kaddar T, Magaud JP, Salles G, and Ffrench M (2011) Cyclin-dependent kinase 1 expression is inhibited by p16(INK4a) at the post-transcriptional level through the microRNA pathway. Oncogene 30: 1880-1891.

Coffman BL, Rios GR, King CD, and Tephly TR (1997) Human UGT2B7 catalyzes morphine glucuronidation. Drug Metab Dispos 25:1-4

Congiu M, Mashford ML, Slavin JL, and Desmond PV (2002) UDP glucuronosyltransferase mRNA levels in human liver disease. Drug Metab Dispos 30:129-134.

Court MH (2010) Interindividual variability in hepatic drug glucuronidation: studies into the role of age, sex, enzyme inducers, and genetic polymorphism using the human liver bank as a model system. Drug Metab Rev 42:209-224.

Dluzen DF, Sun D, Salzberg AC, Jones N, Bushey RT, Robertson GP, and Lazarus P (2014) Regulation of UDP-glucuronosyltransferase 1A1 expression and activity by microRNA 491-3p. J Pharmacol Exp Ther 348:465-477.

Dluzen DF, Sutliff AK, Chen G, Watson CJ, Ishmael FT, and Lazarus P (2016) Regulation of UGT2B expression and activity by miR-216b in liver cancer cell lines. $J$ Pharmacol Exp Ther 359:182-193.

Doench JG and Sharp PA (2004) Specificity of microRNA target selection in translational repression. Genes Dev 18:504-511.

Dong L, Li Y, Han C, Wang X, She L, and Zhang H (2014) miRNA microarray reveals specific expression in the peripheral blood of glioblastoma patients. Int J Oncol $\mathbf{4 5}$ : $746-756$

Fournel-Gigleux S, Jackson MR, Wooster R, and Burchell B (1989) Expression of a human liver cDNA encoding a UDP-glucuronosyltransferase catalysing the glucuronidation of hyodeoxycholic acid in cell culture. FEBS Lett 243:119-122.

Gattolliat CH, Thomas L, Ciafrè SA, Meurice G, Le Teuff G, Job B, Richon C, Combaret V, Dessen P, Valteau-Couanet D, et al. (2011) Expression of miR-487b and miR-410 encoded by $14 \mathrm{q} 32.31$ locus is a prognostic marker in neuroblastoma. Br J Cancer 105:1352-1361.

Gregory PA, Gardner-Stephen DA, Rogers A, Michael MZ, and Mackenzie PI (2006) The caudal-related homeodomain protein $\mathrm{Cdx} 2$ and hepatocyte nuclear factor 1alpha cooperatively regulate the UDP-glucuronosyltransferase $2 \mathrm{~B} 7$ gene promoter. Pharmacogenet Genomics 16:527-536.

Grimson A, Farh KK, Johnston WK, Garrett-Engele P, Lim LP, and Bartel DP (2007) MicroRNA targeting specificity in mammals: determinants beyond seed pairing. Mol Cell 27:91-105.

Guillemette C (2003) Pharmacogenomics of human UDP-glucuronosyltransferase enzymes. Pharmacogenomics $J$ 3:136-158.

Hu DG, Gardner-Stephen D, Severi G, Gregory PA, Treloar J, Giles GG, English DR, Hopper JL, Tilley WD, and Mackenzie PI (2010) A novel polymorphism in a forkhead box A1 (FOXA1) binding site of the human UDP glucuronosyltransferase 2B17 gene modulates promoter activity and is associated with altered levels of circulating androstane-3 $\alpha, 17 \beta$-diol glucuronide. Mol Pharmacol 78:714-722.

$\mathrm{Hu}$ DG and Mackenzie PI (2009) Estrogen receptor alpha, fos-related antigen-2, and c-Jun coordinately regulate human UDP glucuronosyltransferase $2 \mathrm{~B} 15$ and $2 \mathrm{~B} 17$ expression in response to 17 beta-estradiol in MCF-7 cells. Mol Pharmacol 76: $425-439$.

Hu DG and Mackenzie PI (2010) Forkhead box protein A1 regulates UDPglucuronosyltransferase $2 \mathrm{~B} 15$ gene transcription in LNCaP prostate cancer cells. Drug Metab Dispos 38:2105-2109.
Hu DG, Meech R, Lu L, McKinnon RA, and Mackenzie PI (2014a) Polymorphisms and haplotypes of the UDP-glucuronosyltransferase 2B7 gene promoter. Drug Metab Dispos 42:854-862.

Hu DG, Meech R, McKinnon RA, and Mackenzie PI (2014b) Transcriptional regulation of human UDP-glucuronosyltransferase genes. Drug Metab Rev 46:421-458.

Hu DG, Rogers A, and Mackenzie PI (2014c) Epirubicin upregulates UDP glucuronosyltransferase $2 \mathrm{~B} 7$ expression in liver cancer cells via the p53 pathway. Mol Pharmacol 85:887-897.

Innocenti F, Iyer L, Ramírez J, Green MD, and Ratain MJ (2001) Epirubicin glucuronidation is catalyzed by human UDP-glucuronosyltransferase 2B7. Drug Metab Dispos 29:686-692.

Ishii Y, Hansen AJ, and Mackenzie PI (2000) Octamer transcription factor-1 enhances hepatic nuclear factor-1alpha-mediated activation of the human UDP glucuronosyltransferase 2B7 promoter. Mol Pharmacol 57:940-947.

Jin C, Miners JO, Lillywhite KJ, and Mackenzie PI (1993) Complementary deoxyribonucleic acid cloning and expression of a human liver uridine diphosphateglucuronosyltransferase glucuronidating carboxylic acid-containing drugs. $J$ Pharmacol Exp Ther 264:475-479.

Lépine J, Bernard O, Plante M, Têtu B, Pelletier G, Labrie F, Bélanger A and Guillemette $\mathrm{C}$ (2004) Specificity and regioselectivity of the conjugation of estradiol, estrone, and their catecholestrogen and methoxyestrogen metabolites by human uridine diphospho-glucuronosyltransferases expressed in endometrium. $J$ Clin Endocrinol Metab 89:5222-5232.

Lévesque E, Beaulieu M, Hum DW, and Bélanger A (1999) Characterization and substrate specificity of UGT2B4 (E458): a UDP-glucuronosyltransferase encoded by a polymorphic gene. Pharmacogenetics 9:207-216.

Liu D, Hu X, Zhou H, Shi G, and Wu J (2014) Identification of aberrantly expressed miRNAs in gastric cancer. Gastroenterol Res Pract 2014:473817.

Liu S, Guo W, Shi J, Li N, Yu X, Xue J, Fu X, Chu K, Lu C, Zhao J, et al. (2012) MicroRNA-135a contributes to the development of portal vein tumor thrombus by promoting metastasis in hepatocellular carcinoma. J Hepatol 56:389-396.

Livak KJ and Schmittgen TD (2001) Analysis of relative gene expression data using real-time quantitative PCR and the 2(-delta delta C(T)) method. Methods 25: $402-408$

Loureiro AI, Fernandes-Lopes C, Bonifácio MJ, Wright LC, and Soares-da-Silva P (2011) Hepatic UDP-glucuronosyltransferase is responsible for eslicarbazepine glucuronidation. Drug Metab Dispos 39:1486-1494

Lu Y, Heydel JM, Li X, Bratton S, Lindblom T, and Radominska-Pandya A (2005) Lithocholic acid decreases expression of UGT2B7 in Caco-2 cells: a potential role for a negative farnesoid X receptor response element. Drug Metab Dispos 33: 937-946.

Macfarlane LA and Murphy PR (2010) MicroRNA: biogenesis, function and role in cancer. Curr Genomics 11:537-561.

Mackenzie PI, Bock KW, Burchell B, Guillemette C, Ikushiro S, Iyanagi T, Miners JO, Owens IS, and Nebert DW (2005) Nomenclature update for the mammalian UDP glycosyltransferase (UGT) gene superfamily. Pharmacogenet Genomics 15: $677-685$

Mackenzie PI, Owens IS, Burchell B, Bock KW, Bairoch A, Bélanger A, Fournel-Gigleux S, Green M, Hum DW, Iyanagi T, et al. (1997) The UDP glycosyltransferase gene superfamily: recommended nomenclature update based on evolutionary divergence. Pharmacogenetics 7:255-269.

Margaillan G, Lévesque É, and Guillemette C (2016) Epigenetic regulation of steroid inactivating UDP-glucuronosyltransferases by microRNAs in prostate cancer. $J$ Steroid Biochem Mol Biol 155:85-93.

Marrone AK, Shpyleva S, Chappell G, Tryndyak V, Uehara T, Tsuchiya M, Beland FA, Rusyn I, and Pogribny IP (2016) Differentially expressed microRNAs provide mechanistic insight into fibrosis-associated liver carcinogenesis in mice. $\mathrm{Mol} \mathrm{Car}$ cinog 55:808-817.

Maul R, Warth B, Schebb NH, Krska R, Koch M, and Sulyok M (2015) In vitro glucuronidation kinetics of deoxynivalenol by human and animal microsomes and recombinant human UGT enzymes. Arch Toxicol 89:949-960.

Nakamura A, Nakajima M, Higashi E, Yamanaka H, and Yokoi T (2008) Genetic polymorphisms in the 5'-flanking region of human UDP-glucuronosyltransferase 2B7 affect the Nrf2-dependent transcriptional regulation. Pharmacogenet Genomics 18:709-720.

Negrini M, Gramantieri L, Sabbioni S, and Croce CM (2011) microRNA involvement in hepatocellular carcinoma. Anticancer Agents Med Chem 11:500-521.

Ohno A, Saito Y, Hanioka N, Jinno H, Saeki M, Ando M, Ozawa S, and Sawada J (2004) Involvement of human hepatic UGT1A1, UGT2B4, and UGT2B7 in the glucuronidation of carvedilol. Drug Metab Dispos 32:235-239.

Ohno S and Nakajin S (2009) Determination of mRNA expression of human UDPglucuronosyltransferases and application for localization in various human tissues by real-time reverse transcriptase-polymerase chain reaction. Drug Metab Dispos 37:32-40.

Pasquinelli AE (2012) MicroRNAs and their targets: recognition, regulation and an emerging reciprocal relationship. Nat Rev Genet 13:271-282.

Raungrut P, Uchaipichat V, Elliot DJ, Janchawee B, Somogyi AA, and Miners JO (2010) In vitro-in vivo extrapolation predicts drug-drug interactions arising from inhibition of codeine glucuronidation by dextropropoxyphene, fluconazole, ketoconazole, and methadone in humans. J Pharmacol Exp Ther 334:609-618.

Ritter JK, Chen F, Sheen YY, Lubet RA, and Owens IS (1992) Two human liver cDNAs encode UDP-glucuronosyltransferases with $2 \mathrm{log}$ differences in activity toward parallel substrates including hyodeoxycholic acid and certain estrogen derivatives. Biochemistry 31:3409-3414.

Saetrom P, Heale BS, Snøve O, Jr, Aagaard L, Alluin J, and Rossi JJ (2007) Distance constraints between microRNA target sites dictate efficacy and cooperativity. Nucleic Acids Res 35:2333-2342.

Seo KA, Bae SK, Choi YK, Choi CS, Liu KH, and Shin JG (2010) Metabolism of 1'- and 4-hydroxymidazolam by glucuronide conjugation is largely mediated by UDPglucuronosyltransferases 1A4, 2B4, and 2B7. Drug Metab Dispos 38:2007-2013. 
Tang W, Jiang Y, Mu X, Xu L, Cheng W, and Wang X (2014) MiR-135a functions as a tumor suppressor in epithelial ovarian cancer and regulates HOXA10 expression. Cell Signal 26:1420-1426.

Theodore SC, Davis M, Zhao F, Wang H, Chen D, Rhim J, Dean-Colomb W, Turner T, Ji W, Zeng G, et al. (2014) MicroRNA profiling of novel African American and Caucasian prostate cancer cell lines reveals a reciprocal regulatory relationship of miR-152 and DNA methyltransferase 1. Oncotarget 5:3512-3525.

Turgeon D, Carrier JS, Levesque E, Hum DW, and Belanger A (2001) Relative enzymatic activity, protein stability, and tissue distribution of human steroidmetabolizing UGT2B subfamily members. Endocrinology 142:778-787.

Uchaipichat V, Mackenzie PI, Guo XH, Gardner-Stephen D, Galetin A, Houston JB, and Miners JO (2004) Human UDP-glucuronosyltransferases: isoform selectivity and kinetics of 4-methylumbelliferone and 1-naphthol glucuronidation, effects of organic solvents, and inhibition by diclofenac and probenecid. Drug Metab Dispos 32:413-423.

Uchaipichat V, Suthisisang C, and Miners JO (2013) The glucuronidation of R- and S-lorazepam: human liver microsomal kinetics, UDP-glucuronosyltransferase enzyme selectivity, and inhibition by drugs. Drug Metab Dispos 41:1273-1284.

Wang Y, Fu J, Jiang M, Zhang X, Cheng L, Xu X, Fan Z, Zhang J, Ye Q, and Song H (2014) miR-410 is overexpressed in liver and colorectal tumors and enhances tumor cell growth by silencing FHL1 via a direct/indirect mechanism. PLoS One 9: e108708.

Wijayakumara DD, Hu DG, Meech R, McKinnon RA, and Mackenzie PI (2015) Regulation of human UGT2B15 and UGT2B17 by miR-376c in prostate cancer cell lines. J Pharmacol Exp Ther 354:417-425.

Williams JA, Hyland R, Jones BC, Smith DA, Hurst S, Goosen TC, Peterkin V, Koup JR, and Ball SE (2004) Drug-drug interactions for UDP-glucuronosyltransferase substrates: a pharmacokinetic explanation for typically observed low exposure (AUCi/AUC) ratios. Drug Metab Dispos 32:1201-1208

Zeng YB, Liang XH, Zhang GX, Jiang N, Zhang T, Huang JY, Zhang L, and Zeng XC (2016) miRNA-135a promotes hepatocellular carcinoma cell migration and invasion by targeting forkhead box O1. Cancer Cell Int 16:63.

Address correspondence to: Dr. Robyn Meech, Department of Clinical Pharmacology, Flinders University School of Medicine, Flinders Medical Centre, Bedford Park SA 5042, Australia. E-mail: robyn.meech@flinders.edu. au or Dr. Dong Gui, Department of Clinical Pharmacology, Flinders University School of Medicine, Flinders Medical Centre, Bedford Park SA 5042, Australia. E-mail: donggui.hu@flinders.edu.au 